\title{
Lower body positive pressure: an emerging technology in the battle against knee osteoarthritis?
}

This article was published in the following Dove Press journal:

Clinical Interventions in Aging

24 July 2013

Number of times this article has been viewed

\author{
Judit Takacs' \\ Judy E Anderson ${ }^{1,3}$ \\ Jeff RS Leiter ${ }^{1,2,4}$ \\ Peter B MacDonald ${ }^{2,4}$ \\ Jason D Peeler ${ }^{1,4}$ \\ 'Department of Human Anatomy and \\ Cell Science, ${ }^{2}$ Department of Surgery, \\ ${ }^{3}$ Department of Biological Sciences, \\ University of Manitoba, Winnipeg, \\ Manitoba, Canada; ${ }^{4}$ Pan Am Clinic, \\ Winnipeg, Manitoba, Canada
}

Background: Knee osteoarthritis (OA) is the most prevalent medical condition in individuals over the age of 65 years, and is a progressive joint degenerative condition with no known cure. Research suggests that there is a strong relationship between knee pain and loss of physical function. The resulting lifestyle modifications negatively impact not only disease onset and progression but also overall health, work productivity, and quality of life of the affected individual.

Purpose: The goal of this investigation was to examine the feasibility of using an emerging technology called lower body positive pressure (LBPP) to simulate weight loss and reduce acute knee pain during treadmill walking exercise in overweight individuals with radiographically confirmed symptomatic knee OA.

Design: Prospective case series.

Methods: Twenty-two overweight individuals with knee OA completed two 20-minute treadmill walking sessions (one full weight bearing and one LBPP supported) at a speed of $3.1 \mathrm{mph}$, $0 \%$ incline. Acute knee pain was assessed using a visual analog scale, and the percentage of LBPP support required to minimize knee pain was evaluated every 5 minutes. Knee Osteoarthritis Outcome Scores were used to quantify knee pain and functional status between walking sessions. The order of testing was randomized, with sessions occurring a minimum of 1 week apart.

Results: A mean LBPP of $12.4 \%$ of body weight provided participants with significant pain relief during walking, and prevented exacerbation of acute knee pain over the duration of the 20-minute exercise session. Patients felt safe and confident walking with LBPP support on the treadmill, and demonstrated no change in Knee Osteoarthritis Outcome Scores over the duration of the investigation.

Conclusion: Results suggest that LBPP technology can be used safely and effectively to simulate weight loss and reduce acute knee pain during weight-bearing exercise in an overweight knee OA patient population. These results could have important implications for the development of future treatment strategies used in the management of at-risk patients with progressive knee OA.

Keywords: knee osteoarthritis, knee pain, exercise, lower body positive pressure

\section{What is known about the subject?}

This investigation is the first to examine the feasibility of using a new and emerging technology called lower body positive pressure (LBPP) to support low-load treadmill walking exercise in an at-risk knee osteoarthritis (OA) patient population.

Correspondence: Jason D Peeler Department of Human Anatomy and Cell Science, Faculty of Medicine, University of Manitoba, 102-745 Bannatyne Ave, Winnipeg, Manitoba, Canada

Tel +l 2042723 | 46

Email peelerj@cc.umanitoba.ca

\section{What this study adds to the existing knowledge}

LBPP is an emerging unweighting technology that can be used safely and successfully to simulate weight loss and study low-load weight-bearing exercise in overweight 
patients with progressive knee OA. LBPP support of only $12.4 \%$ body weight was required to manage and prevent exacerbation of acute knee pain symptoms during treadmill walking exercise.

\section{Introduction}

Knee OA is the most common form of arthritis, ${ }^{1}$ currently affecting more than 25 million North Americans, with the incidence expected to double by the year $2020 .{ }^{1,2}$ In fact, the current rate of knee $\mathrm{OA}$ is as high as that of cardiac disease, and it is the most prevalent medical condition in individuals aged over 65 years. ${ }^{3}$ It is a joint pathology characterized by the formation of osteophytes and cysts, narrowed joint spacing, and subchondral bone sclerosis. ${ }^{4}$ Although the age of onset and symptoms related to joint degeneration can vary greatly from patient to patient, disease progression is commonly associated with progressive and debilitating joint pain, stiffness, muscle weakness, and decreased joint range of motion. These signs and symptoms make it difficult to perform essential activities of daily living such as walking, squatting, and going up and down stairs. ${ }^{5}$ Implications of disease progression include restrictions in daily activities, reduced work productivity, and diminished quality of life. ${ }^{2,6}$

At present, there is no known cure. ${ }^{7}$ Current approaches to nonoperative treatment primarily focus on the management of symptoms through the use of pharmacological interventions (such as analgesic and nonsteroidal anti-inflammatory medications) that are designed to target joint pain and swelling associated with disease progression, or through nutritional supplementation (such as chondroitin and glucosamine) that is postulated to improve articular cartilage health. ${ }^{8}$ Unfortunately, these passive forms of intervention do very little to address underlying risk factors (such as obesity, abnormal joint kinematics due to injury, thigh muscle weakness, and leg misalignment) that have been clearly identified within the OA literature as having a direct impact on the age of onset and rate of disease progression, ${ }^{9}$ and in many instances may actually place the patient at significant risk for the development of other comorbidities (eg, gastrointestinal bleeding and renal and cardiac disease). ${ }^{10,11}$

Of the identified risk factors, being overweight (body mass index $\left.[\mathrm{BMI}]>25 \mathrm{~kg} / \mathrm{m}^{2}\right)^{12-14}$ is believed to be the number one modifiable risk factor for the development and progression of knee OA. ${ }^{1}$ Multiple studies have demonstrated that high body weight precedes the development of knee OA, ${ }^{15,16}$ influences the age of onset and rate of disease progression, ${ }^{17-19}$ quadruples the risk of developing knee
OA for both genders, ${ }^{20}$ and increases the risk for developing $\mathrm{OA}$ in the contralateral knee. ${ }^{1,21}$ Research examining the relationship between weight loss and joint function in a knee OA patient population illustrates that even small amounts of weight loss (as little as 5\% over an 18-month period in overweight patients) can lead to an improvement in subjective reporting of joint pain and function. ${ }^{22}$ To date, researchers have been unable to quantify the strength of the relationship between loss of body weight and change in knee joint symptoms ${ }^{23}$ (ie, is it a linear relationship?), little information is available to guide individualized prescription of weight loss for the management of joint pain and dysfunction, and the specific impact that weight loss has on knee joint kinematics during walking within a knee OA patient population is unknown.

Knee OA research also illustrates that weight-bearing exercise such as a daily walking regimen is effective for managing joint symptoms, enhancing functional capacity and quality of life, and decreasing patient reliance on analgesic medication. ${ }^{5,24}$ As a result, regular exercise is a recommended treatment strategy by both the American College of Rheumatology and the European League Against Rheumatism. ${ }^{8,25}$ When one considers that a force of roughly three to six times body weight is exerted across a healthy knee joint during the single stance phase of walking, it becomes clear that an overweight or obese person with knee OA is at significant risk for exacerbation of joint symptoms and disease progression when initiating a regular weight-bearing walking program. ${ }^{26}$

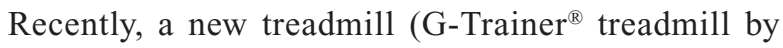
AlterG Inc, Fremont, CA, USA) (Figure 1) was introduced that permits low-load walking using an emerging technology called LBPP. ${ }^{27}$ The system utilizes a waist-high air chamber

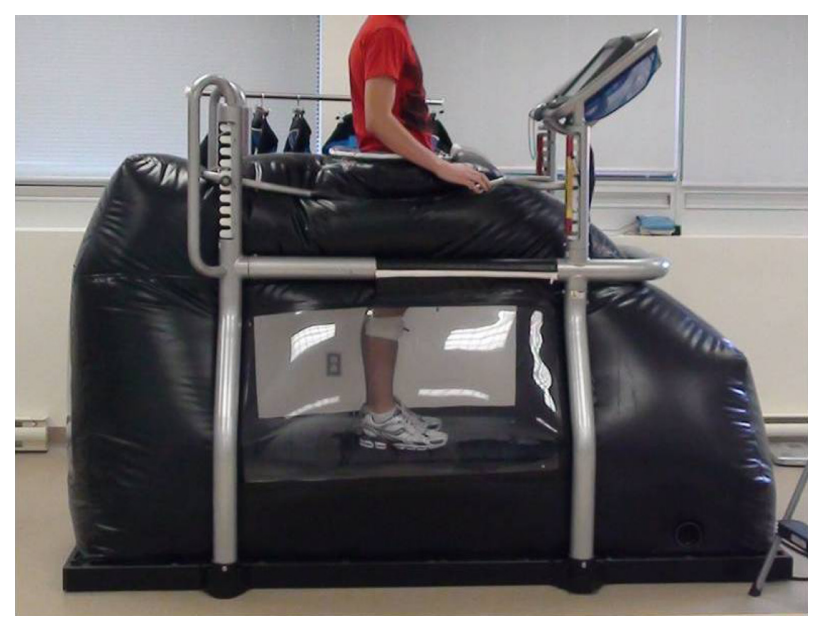

Figure I Subject walking on a G-Trainer treadmill under lower body positive pressure support. 
that can be inflated with positive air pressure in order to modify body weight during ambulation. During an exercise session, subjects wear a pair of neoprene shorts with a kayakstyle skirt that zips into an air chamber, creating an airtight seal. When the air chamber inflates, there is an increase in air pressure around the lower body that lifts the subject upwards at the hips. ${ }^{27,28}$ This effectively reduces body weight and the gravitational forces about the lower extremity to a level that can be adjusted with a high degree of consistency. ${ }^{28,29}$ Positive air pressure can then be used to accurately unweight a person by increments as small as $1 \%$ body weight and as large as $80 \%$ body weight. ${ }^{30} \mathrm{LBPP}$ is recognized as being superior to other methods of unweighting (such as an upper body harness that partially supports body weight, or aquatic exercises), because the air pressure is applied uniformly over the lower body, thus reducing the formation of pressure points that are common with harness-based systems, ${ }^{31,32}$ while maintaining normal muscle activation and gait patterns (which are altered during aquatic-based activities). ${ }^{28,33,34}$ As a result, the LBPP treadmill technology is gaining popularity as a device that offers the ability to study weight-supported or low-load exercise in a user-friendly and kinematically correct manner, ${ }^{28,35}$ without altering gait dynamics ${ }^{28}$ or cardiovascular parameters such as heart rate and blood pressure. ${ }^{29,36}$ LBPP trials have so far been reported on several musculoskeletal conditions, including meniscectomy and anterior cruciate ligament reconstruction, ${ }^{27}$ as well as lower limb trauma. ${ }^{37}$ Each of these studies used LBPP to create a low-load exercise regime that essentially permitted patients to resume early and/or pain-free ambulation, or to walk further and for longer periods than was previously possible during normal walking (ie, during full weight bearing [FWB]) without exacerbation of symptoms. These reports suggest LBPP may hold promise as a method of providing a remediated weight-bearing exercise regimen for those with progressive knee OA or those at risk for developing or exacerbating knee OA symptoms due to obesity that precludes normal exercise activities. To date, the use of this emerging technology with an overweight knee OA patient population has gone unreported within the literature, and the feasibility of utilizing the LBPP technology to artificially simulate weight loss and facilitate pain-free ambulatory exercise in this patient population is unknown.

\section{Purpose of the study}

The purpose of this study was to investigate the feasibility of using LBPP support to simulate weight loss and manage acute knee pain during treadmill walking exercise in overweight individuals with radiographically confirmed symptomatic knee OA. The hypothesis was that LBPP support would significantly reduce acute knee pain during treadmill walking exercise in overweight knee OA patients.

\section{Methods Setting and participants}

Following ethics board review and approval by the local regional health authority, 22 overweight participants $\left(\mathrm{BMI}>25 \mathrm{~kg} / \mathrm{m}^{2}\right)$ between the ages of 35 and 60 years, with radiographically confirmed symptomatic mild to moderate knee OA were recruited to participate in the study. Each participant was assigned an identification number and required to complete informed consent, personal information, and knee demographic forms prior to initiation of participation in the study. The knee demographic form provided a detailed history of the patients' knee OA, such as previous history of injury or surgery, and previous and current treatment methods, such as bracing, injections, and pharmacological intervention. In addition, the form was also used to confirm knee symptoms, knee function, and activities that initiate knee pain symptoms, as well as to provide information relevant to the inclusion/ exclusion criteria. Participants were excluded if they had a recent history of traumatic hip, knee, or ankle surgery (within the last year), had severe knee OA, or were diagnosed with rheumatoid arthritis, chronic reactive arthritis, or psoriatic arthritis. Anteroposterior radiographs were taken for all subjects in a weight-bearing position with both knees in $5^{\circ}$ of flexion. Each radiograph was reviewed by a senior radiologist specializing in musculoskeletal imaging, and scored using the Kellgren-Lawrence scale, ${ }^{38}$ with subjects who scored 0 (indicating no signs of OA) and 4 (indicating severe OA) being excluded from participation. ${ }^{1,8}$ Participants also completed a short questionnaire to assess health-enhancing physical activity (SQUASH questionnaire), ${ }^{39}$ and anthropometric measurements such as height and weight were taken prior to the first treadmill walking session and then used to calculate BMI. All participants were directed to wear shorts and T-shirts for their assessment, and asked to refrain from exercise a minimum of 4 hours prior to each of their data collection sessions.

\section{Treadmill walking sessions}

All participants completed two G-Trainer treadmill walking sessions during the course of the study, one FWB walking session and one LBPP-supported walking session, which occurred a minimum of 7 days apart. The study utilized a methodology that randomized the order of treadmill testing (FWB or LBPP supported), visually blinded subjects to the 
percentage of LBPP support utilized during each walking session, and ensured that the LBPP blower and air chamber were on and pressurized even when participants were performing the FWB treadmill walking sessions with 0\% LBPP support. Prior to each walking session (as well as 1 week after completing the second walking session), participants completed a Knee Injury and Osteoarthritis Outcome Score (KOOS) questionnaire to quantify knee pain and function over the previous 7-day period. Scoring on the KOOS can range from 0 (complete disability) to 100 (no disability) and is evaluated using five categories: (1) pain, (2) symptoms, (3) function in daily living, (4) sports/recreation, and (5) quality of life. ${ }^{40,41}$ For all treadmill walking sessions, participants were required to walk at a speed of $1.4 \mathrm{~m} / \mathrm{s}$ (3.1 mph) at $0^{\circ}$ incline for a period of 20 minutes.

Each participant completed an initial 5-minute warm-up on the treadmill prior to the initiation of data collection. This allowed subjects to gradually reach the predetermined walking speed (3.1 mph at $0^{\circ}$ incline) and adjust to walking on the treadmill's belt surface. For the FWB walking sessions, no LBPP support was applied (but the LBPP blower and air chamber were on and the chamber pressurized). For the LBPP-supported walking sessions, the goal was to select a percentage of unweighting that completely eliminated or substantially reduced the participants' acute knee pain during treadmill walking. LBPP pressure was constantly monitored and systematically adjusted by $5 \%$ increments (up to a maximum of $30 \%$ LBPP support) to maintain a pain-free walking experience. Visual analog scale (VAS) acute knee pain measures were taken at 5-minute intervals during each treadmill walking session, with the four VAS measures (at minutes 5, 10, 15, and 20) for each walking session averaged in order to obtain an overall "acute knee pain score" for that specific walking session. Participants were blinded to their KOOS and VAS scores from the previous walking session.

\section{Data analysis}

Microsoft Office Excel 2007 with Data Analysis ToolPak add-in (Microsoft Corporation, Redmond, WA, USA) and SPSS Version 17 (IBM Corporation, Armonk, NY, USA) were used for data analysis. The sample size selected for the study was based on a VAS minimum clinically significant difference of $13 / 100 \mathrm{~mm}$, with a standard deviation (SD) of $19 \mathrm{~mm} .{ }^{15}$ Using an $\alpha=0.05$ (two-tailed) and $90 \%$ power level, a total of 23 participants were required for the study $(\mathrm{N}=22)$. Repeated-measures analysis of variance (ANOVA) was used to compare KOOS between sessions 1 and 2 and 1 week postwalking, consecutive VAS knee pain measures taken at 5-minute intervals during either FWB or LBPP-supported treadmill walking sessions, and total (or cumulative) VAS acute knee pain scores between FWB and LBPP-supported treadmill walking during the entire 20-minute walking sessions. $t$-tests were used to compare VAS acute knee pain scores between FWB and LBPP-supported walking at each 5-minute interval during the treadmill walking sessions. Differences were considered statistically significant if $P<0.05$.

\section{Results}

Descriptive data for study participants are summarized in Table 1. Mean ( \pm SD) age and BMI of the 22 participants (17 female; five male) were $52.9( \pm 5.9)$ years and $33.6( \pm 6.4) \mathrm{kg} / \mathrm{m}^{2}$, respectively. Knee demographic data illustrated that eleven of the 22 participants had a history of traumatic knee injury, with the average duration of OA symptoms being $147( \pm 185)$ months among the group. Nine of 22 participants demonstrated bilateral knee OA, with data for the more painful knee being used for statistical analysis. All participants, except one, exhibited medial knee compartment degeneration on radiograph (one exhibited lateral compartment degeneration). Data from the SQUASH physical activity questionnaire indicated that study participants reported high levels of physical activity, spending an average of 9.1 hours per week in physical activity, of which $15.5 \%$ was classified as moderately intense activity and $11.9 \%$ was high-intensity activity.

LBPP-supported walking session data are presented in Table 2. On average, approximately $12.4 \%( \pm 7.4 \%)$ of LBPP support was required in order to minimize participants' knee pain during the LBPP-supported walking session, with 15\% of LBPP support being the median amount of unweighting required to minimize or eliminate acute knee pain over the duration of the 20 -minute walking session, and the maximum

Table I Descriptive information for the sample patient population $^{\mathrm{a}}$

\begin{tabular}{ll}
\hline Participants & $\begin{array}{l}\text { Total (mean } \pm \text { standard } \\
\text { deviation) }\end{array}$ \\
\hline Number & $22(5$ male/ 17 female $)$ \\
Age (years) & $52.9 \pm 6.4$ \\
Height $(\mathrm{m})$ & $1.67 \pm 0.1$ \\
Weight $(\mathrm{kg})$ & $93.7 \pm 18.5$ \\
Body mass index $\left(\mathrm{kg} / \mathrm{m}^{2}\right)$ & $33.6 \pm 6.5$ \\
Leg alignment $(\mathrm{Var} / \mathrm{N} / \mathrm{Val})$ & $3 / 1 / 18$ \\
Physical activity levels $(\mathrm{min} /$ week) & $711 \pm 574$
\end{tabular}

Note: ${ }^{2}$ Values are representative of a homogenous sample representative of an overweight/obese early-onset knee osteoarthritis patient population.

Abbreviations: $\mathrm{N}$, neutral; Val, valgus; Var, varus. 
Table 2 Percentage of support required to minimize acute knee pain during lower body positive pressure (LBPP)-supported walking

\begin{tabular}{ll}
\hline $\begin{array}{l}\text { Walking time } \\
(\mathbf{N}=\mathbf{2 2})\end{array}$ & $\begin{array}{l}\text { Percentage of LBPP support } \\
\text { Mean } \pm \text { standard deviation } \\
\text { (range) }\end{array}$ \\
\hline 5 mins & $\begin{array}{l}12.3 \pm 7.5 \\
(5-30)\end{array}$ \\
10 mins & $12.3 \pm 7.5$ \\
& $(5-30)$ \\
15 mins & $12.6 \pm 7.5$ \\
& $(5-30)$ \\
20 mins & $12.6 \pm 7.5$ \\
& $(5-30)$ \\
Total & $12.4 \pm 7.4$ \\
\hline
\end{tabular}

LBPP support ranging from $5 \%$ to $30 \%$ of total body weight among participants.

VAS knee pain data collected at 5-minute intervals during both 20-minute walking sessions (FWB and LBPP supported) are summarized in Table 3. VAS scoring for knee pain during the LBPP walking session was consistently lower than VAS scoring for the FWB walking session at each 5-minute interval (however, these differences were not statistically different), with the size of the difference in pain scores increasing as the duration of the walking session became longer. However, repeated-measures ANOVA testing revealed a statistically significant difference $(P<0.05)$ only when comparing the total (or cumulative) "acute knee pain score" from all VAS measurements (ie, average of VAS measures taken at minutes 5, 10, 15, and 20) taken during each LBPP and FWB 20-minute walking session.

Figure 2 further illustrates the cumulative treatment effect associated with LBPP-supported walking.

Table 3 Acute knee pain during walking ${ }^{\mathrm{a}}$

\begin{tabular}{lll}
\hline $\begin{array}{l}\text { Walking time } \\
(\mathbf{N}=\mathbf{2 2})\end{array}$ & $\begin{array}{l}\text { LBPP walking } \\
\text { Mean } \pm \mathbf{S D}\end{array}$ & $\begin{array}{l}\text { FWB walking } \\
\text { Mean } \pm \mathbf{S D}\end{array}$ \\
\hline 5 mins & $19.6 \pm 18.6$ & $24.6 \pm 16.5$ \\
& $(11.4-27.8)$ & $(17.3-31.9)$ \\
$10 \mathrm{mins}$ & $21.5 \pm 20.5$ & $27.9 \pm 17.8$ \\
& $(12.4-30.6)$ & $(20.0-35.8)$ \\
15 mins & $23.2 \pm 23.0$ & $31.4 \pm 21.5$ \\
& $(12.7-33.7)$ & $(21.8-40.9)$ \\
20 mins & $26.4 \pm 24.1$ & $33.9 \pm 24.3$ \\
& $(15.1-37.7)$ & $(23.1-44.6)$ \\
Total & $22.6 \pm 21.5$ & $30.0 \pm 20.1 *$ \\
\hline
\end{tabular}

Notes: a Comparison of participant knee pain (mean \pm standard deviation [SD]) and confidence intervals $(95 \% \mathrm{Cl})$ as measured by the visual analog scale $(\mathrm{mm})$ for 20 minutes of treadmill walking (recorded at 5-minute intervals) under lower body positive pressure (LBPP)-supported and full weight-bearing (FWB) walking conditions. $* P<0.05$.
Baseline (mean \pm SD) VAS pain scores for LBPP and FWB walking sessions were $21.2 \pm 17.5$ and $20.9 \pm 17.1$, respectively. Over the course of the 20-minute walking session, VAS pain levels for the LBPP-supported walking sessions initially dropped, and then increased only marginally. In comparison, VAS pain levels for the FWB walking session steadily increased over the duration of the 20 -minute walking session. The knee pain experienced during FWB walking increased significantly between the first pain data point (at the 5-minute interval) and the last pain data collection point (at the 20-minute interval) ( $P=0.002$ ), with pain increases at each 5-minute interval being statistically significant. Importantly, knee pain did not significantly increase over the entire duration of the LBPP-supported treadmill walking sessions $(P=0.58)$ or between any of the individual 5-minute intervals.

Knee function as measured on the KOOS is summarized in Table 4. Response rates for the first two surveys were $100 \%$, and $65.0 \%$ for the last survey. Missing data from individual sections of the KOOS were calculated using methods previously described in the literature. ${ }^{41}$ Briefly, if scoring on one or two questions was missing in a specific subsection, the average for that subsection was substituted as the score for that specific question. If more than two values were missing, the subscale score was considered invalid and was not calculated. There were no cases of more than two values missing per subscale. Analysis via repeated-measures ANOVA indicated that KOOS scoring remained constant throughout participants' involvement in the study, with no significant differences being noted when comparing the results of the first, second, and third survey.

\section{Discussion}

To our knowledge, this investigation is the first to examine the feasibility of using a new and emerging technology called LBPP to support low-load treadmill walking exercise in an at-risk knee OA patient population. The results suggest that LBPP support can be utilized safely and successfully to simulate weight loss and manage acute knee pain during prolonged walking exercise. LBPP support of only $12.4 \%$ body weight was required to significantly diminish knee pain in overweight knee OA patients. These results could have important implications for the development of future treatment strategies used in management of joint pain and dysfunction associated with the progressive knee OA in at-risk patients.

The individuals who participated in this investigation were representative of a young (age range 36-64 years) and overweight (range $25-47 \mathrm{~kg} / \mathrm{m}^{2}$ ) patient population with 


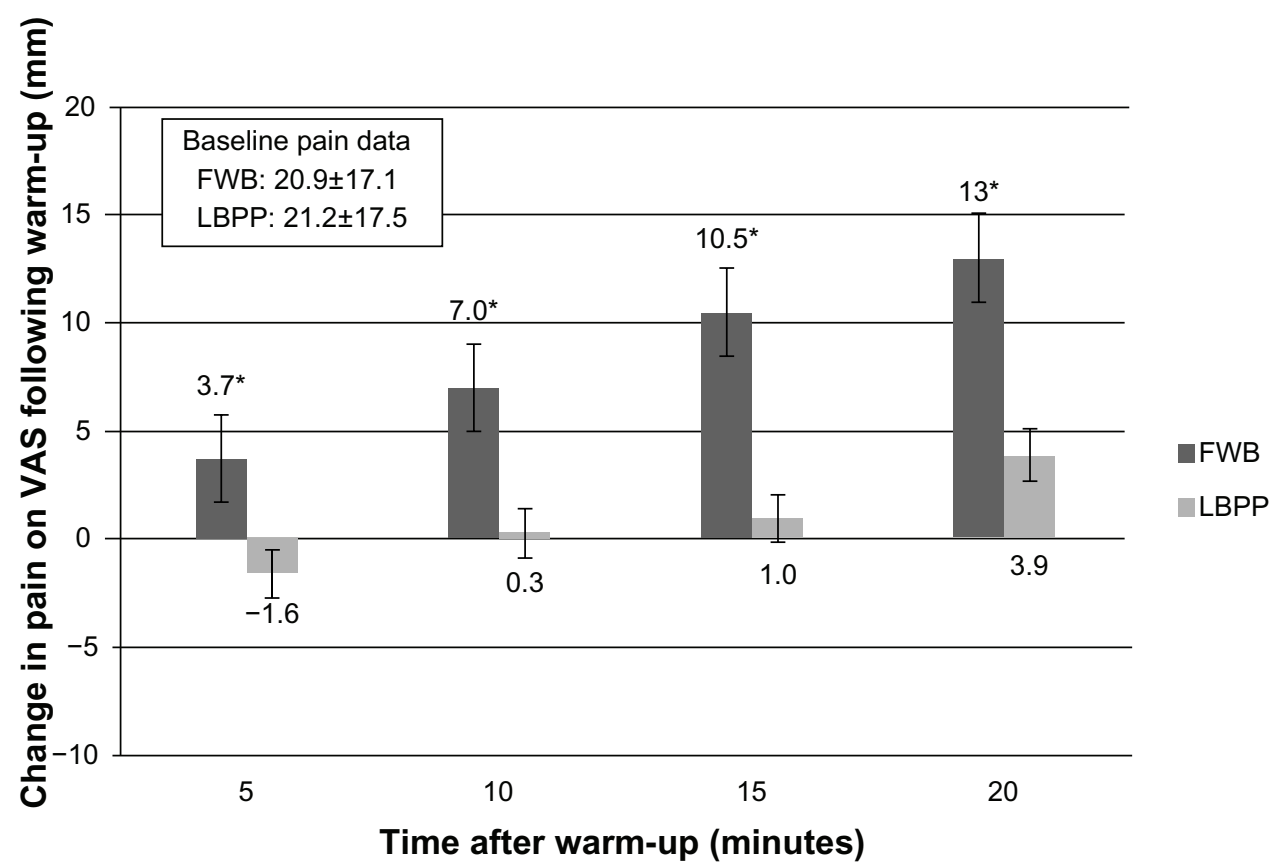

Figure 2 Change in knee pain during walking. ${ }^{a}$

Notes: 'Mean change in pain as measured on the visual analog scale (VAS) during treadmill walking and expressed relative to the patients' baseline pain score (which was representative of zero). Over the four intervals, pain increased significantly in the full weight-bearing (FWB) condition (*P=0.002; 95\% confidence interval 5.3-20.7) but not in the lower body positive pressure (LBPP) condition ( $P=0.58$; $95 \%$ confidence interval $-16.0-9.2$ ).

early-onset knee OA. Patients reported experiencing progressive symptoms of joint pain, stiffness, muscle weakness, and decreased joint range of motion for more than 10 years (on average), with radiographic evaluation of the affected joint revealing the presence of joint space narrowing and osteophyte formation for all participants. KOOS for knee pain and function were consistent over the duration of the investigation and comparable with previous reports examining a knee OA patient population. Interestingly, physical activity data (from the SQUASH survey) revealed that although individuals still participated in activities that would be classified as "moderate or vigorous physical activity," such as sporting

Table 4 Knee Injury and Osteoarthritis Outcome Score (KOOS) scoring

\begin{tabular}{|c|c|c|c|}
\hline \multirow[t]{2}{*}{$\begin{array}{l}\text { KOOS } \\
\text { category }\end{array}$} & $\begin{array}{l}\text { Walking } \\
\text { session I }\end{array}$ & $\begin{array}{l}\text { Walking } \\
\text { session } 2\end{array}$ & $\begin{array}{l}\text { One week } \\
\text { postwalking }\end{array}$ \\
\hline & Mean \pm SD & Mean \pm SD & Mean \pm SD \\
\hline Pain & $48.9 \pm 16.0$ & $51.0 \pm 15.9$ & $54.6 \pm 16.6$ \\
\hline Symptoms & $48.2 \pm 17.5$ & $46.2 \pm 16.6$ & $53.6 \pm 17.6$ \\
\hline $\begin{array}{l}\text { Function in daily } \\
\text { living }\end{array}$ & $59.5 \pm 20.8$ & $56.9 \pm 17.9$ & $63.2 \pm 20.1$ \\
\hline Sports/recreation & $22.3 \pm 23.0$ & $23.7 \pm 18.1$ & $29.2 \pm 27.9$ \\
\hline Quality of life & $28.4 \pm 22.1$ & $29.3 \pm 17.6$ & $35.9 \pm 20.3$ \\
\hline
\end{tabular}

Note: ${ }^{\mathrm{K} O O O S}$ data were consistent with previous reports for a knee osteoarthritis patient population, with no significant changes in KOOS scoring being noted between any of the three data collection periods.

Abbreviation: SD, standard deviation. and recreational activities (ie, activities requiring energy expenditures greater than four metabolic equivalents), KOOS scoring suggested that they experienced significant difficulties during these types of participation, and that this had a negative impact on their overall quality of life scores. This finding may be reflective of the fact that the study sample was representative of a young knee OA demographic who still regularly participated in sporting and recreational activities despite the risk of further exacerbation of knee pain and joint symptoms. Although OA is often thought of as a disease of the elderly, the incidence of this degenerative condition at younger ages is becoming more prevalent, with approximately $5 \%$ of the population aged 35-54 years presenting with radiographic signs of knee OA. ${ }^{42}$ Research demonstrates that adolescents and young adults with a history of traumatic joint injury, especially at the knee, are at greater than five-fold increased risk for the development of future OA. ${ }^{43-46}$

Of the identified risk factors, being overweight (BMI of $>25 \mathrm{~kg} / \mathrm{m}^{2}$ ) is considered to be the number one modifiable risk factor for the development and progression of knee OA, with research suggesting that each $1 \mathrm{lb}(0.45 \mathrm{~kg})$ of weight gain results in a corresponding $4 \mathrm{lb}(1.81 \mathrm{~kg})$ increase in knee joint loads during walking. ${ }^{47}$ If high body weight results in an increased risk in the onset and progression of knee OA, then decreasing body weight may have preventive effects. In the current study, an emerging technology called LBPP was 
successfully used to simulate weight loss and diminish acute knee joint pain and permit low-load walking exercise in a young overweight knee OA patient population. Data indicated that an average LBPP support of only $12.4 \%$ ( $\pm 7.4 \%)$ of body weight resulted in a significant decrease in a participant's acute knee pain while treadmill walking for 20 minutes. This finding is significant for several reasons.

First, our study confirms the findings of previous investigations that have indicated that LPBB-supported exercise interventions are a safe and user-friendly method of remediating weight-bearing exercise. ${ }^{37}$ Previous reports have examined the use of the LBPP technology in the rehabilitation of young and otherwise healthy, physically active patients who have sustained acute musculoskeletal injuries. ${ }^{27,37}$ Each of these investigations utilized the LBPP technology as a method to successfully remediate or accelerate a progressive rehabilitation protocol, with the level of LBPP support, walking speed, and/or incline being adjusted as the patient progressed through their rehabilitation. Our investigation is the first to examine the feasibility of LBPP support in the management of a chronic or degenerative musculoskeletal condition, utilizing a standardized methodological approach (ie, 5-minute warm-up, 20 minutes of treadmill walking at $3.1 \mathrm{mph}, 0 \%$ incline, $60 \%-80 \%$ maximal heart rate). All participants were able to complete the prescribed treadmill protocol under both the FWB and LBPP-supported walking conditions, with no participants withdrawing from the study. The consistency of KOOS data suggests that participants did not experience any exacerbation of joint symptoms over the duration of the investigation, that LBPP pressure was well tolerated, and that the prescribed walking protocol facilitated safe and user-friendly weight-bearing exercise.

Second, to our knowledge, this investigation represents the first published report on the feasibility of utilizing the LBPP technology to simulate weight loss and control acute knee pain in an overweight knee OA patient population during walking exercise. Results indicate that LBPP support can be used to significantly control acute knee pain during treadmill walking, and unlike FWB walking, LBPPsupported walking did not result in a progressive and significant increase in acute pain symptoms over the duration of a 20-minute treadmill walk. This suggests that the LBPP technology may hold promise as a rehabilitative tool that can be utilized to successfully remediate weight-bearing exercise intervention strategies commonly used in the treatment of lower-extremity pathologies such as knee OA.

Finally, and possibly most importantly, the LBPP technology allowed for patient-specific feedback regarding acute knee pain to determine the percentage of unweighting required to manage acute knee pain during prolonged walking exercise. Anecdotally, patients commented that the LBPP support allowed them to walk pain free for the first time in years, and others indicated that they were surprised about the positive impact that air support could have on their knee pain symptoms. This approach facilitated more accuracy in the treatment intervention, and allowed the relationship between body weight and acute knee pain to be quantified in a patient-specific manner. The results indicated that the percentage of LBPP support required to minimize or eliminate knee pain during weight-bearing exercise was representative of a realistic and attainable level of weight loss for an overweight person with progressive knee OA. Prior to this investigation, no information was available regarding the percentage of LBPP required to attenuate joint pain in an overweight knee OA patient population during a steady-state exercise condition. When placed in a body weight context, the mean percentage of LBPP support required to manage acute knee pain during walking simulated a decrease in participants' body weight of $11.6 \mathrm{~kg}$ (mean body weight of $93.7 \mathrm{~kg} \times 12.4 \%=11.6 \mathrm{~kg}$ ), thus representing a reduction of participants' average BMI from $33.6 \mathrm{~kg} / \mathrm{m}^{2}$ (which placed them in the categorization of obese) to $29.4 \mathrm{~kg} / \mathrm{m}^{2}$ (reducing their categorization to overweight).

There are several limitations to the current study. This study utilized two isolated treadmill walking sessions to examine the feasibility of using LBPP support to simulate weight loss and enable pain-free low-load walking exercise in overweight knee OA patients. The efficacy of LBPP support for the modulation of knee pain in a chronic exercise/ rehabilitation setting is still unknown, and caution should be used when interpreting these results as they relate to the design of future exercise interventions targeting a knee OA population. Instead, further long-term research is needed to clarify the role that this emerging unweighting technology could play in the management of joint symptoms associated with progressive knee OA in an overweight patient population. This investigation also utilized a within-subject design to look at changes in knee pain and function, with the FWB walking session serving as the control condition. There was no healthy control group used to compare changes in knee pain and function. Beyond this, concerns about how a patient's physical perception of LBPP support (ie, the air pressure) may confound VAS scoring of knee pain are valid (ie, a patient who perceives higher LBPP support may score their acute knee pain during walking lower on the VAS scale). 
Finally, the question of whether this type of exercise intervention is cost-effective or superior to other methods of low-impact, non-weight-bearing exercise warrants further investigation. Although there is some evidence to suggest that task-specific activities such as walking are superior to other non-weight-bearing activities such as strength training, cycling, and aquatic exercise ${ }^{5,26,48}$ (because of its ability to promote and/ or restore normal muscle strength, joint proprioception, and the joint range of motion necessary to effectively perform typical activities of daily living), research examining exercise regimens used in the management of knee OA has led to only general exercise recommendations or guidelines, with little evidence available to support the efficacy of patient or symptom-specific manipulation of the exercise regimen being prescribed. ${ }^{8,49,50}$ Beyond this, although some investigations have attempted to compare the impact of different forms of exercise on knee pain and function in a knee OA population, ${ }^{26,50,51}$ research comparing the treatment/cost efficacy of these different forms of exercise is currently lacking.

\section{Conclusion}

The purpose of this investigation was to examine the feasibility of using an emerging technology called LBPP to simulate weight loss and manage acute knee pain during treadmill walking exercise in overweight individuals with radiographically confirmed symptomatic knee OA. Results suggest that this unweighting technology can be used safely and successfully to simulate weight loss in a symptom-specific manner, and that LBPP support of only $12.4 \%$ body weight is required to prevent exacerbation and manage acute knee pain during prolonged treadmill walking exercise. Future research should be directed at exploring how LBPP-supported low-load exercise can be utilized to enhance the functional capacity of knee OA patients, promote physical activity levels and long-term exercise adherence, and slow disease progression in a knee OA patient population.

\section{Acknowledgments}

Operating funds for this research were provided by the Dr Paul HT Thorlakson Foundation and a graduate fellowship (to JT) from the Canadian Institutes of Health Research. Ongoing research support was provided by both the Pan Am Clinic Foundation and the Faculty of Medicine at the University of Manitoba, Canada.

\section{Ethics approval}

This investigation received university ethics board approval and was authorized by the local regional health authority.

\section{Disclosure}

The authors have no conflict of interest to report, and received no financial aid from the equipment manufacturer. We certify that no party having a direct interest in the results of this investigation has or will confer a benefit on us or on any organization with which we are associated.

\section{References}

1. Arden N, Nevitt MC. Osteoarthritis: epidemiology. Best Pract Res Clin Rheumatol. 2006;20(1):3-25.

2. Jinks C, Jordan K, Croft P. Osteoarthritis as a public health problem: the impact of developing knee pain on physical function in adults living in the community: (KNEST 3). Rheumatology (Oxford). 2007;46(5):877-881.

3. Guccione AA, Felson DT, Anderson JJ, et al. The effects of specific medical conditions on the functional limitations of elders in the Framingham Study. Am J Public Health. 1994;84(3):351-358.

4. Luyten FP, Denti M, Filardo G, Kon E, Engebretsen L. Definition and classification of early osteoarthritis of the knee. Knee Surg Sports Traumatol Arthrosc. 2012;20(3):401-406.

5. Evcik D, Sonel B. Effectiveness of a home-based exercise therapy and walking program on osteoarthritis of the knee. Rheumatol Int. 2002;22(3):103-106.

6. Laslett LL, Quinn SJ, Winzenberg TM, Sanderson K, Cicuttini F, Jones G. A prospective study of the impact of musculoskeletal pain and radiographic osteoarthritis on health related quality of life in community dwelling older people. BMC Musculoskelet Disord. 2012;13:168.

7. Rogers MW, Wilder FV. The association of BMI and knee pain among persons with radiographic knee osteoarthritis: a cross-sectional study. BMC Musculoskelet Disord. 2008;9:163.

8. Zhang W, Moskowitz RW, Nuki G, et al. OARSI recommendations for the management of hip and knee osteoarthritis, Part II: OARSI evidence-based, expert consensus guidelines. Osteoarthritis Cartilage. 2008;16(2):137-162.

9. Shrader MW, Draganich LF, Pottenger LA, Piotrowski GA. Effects of knee pain relief in osteoarthritis on gait and stair-stepping. Clin Orthop Relat Res. 2004;(421):188-193.

10. Losina E, Walensky RP, Reichmann WM, et al. Impact of obesity and knee osteoarthritis on morbidity and mortality in older Americans. Ann Intern Med. 2011;154(4):217-226.

11. Denisov LN, Nasonova VA, Koreshkov GG, Kashevarova NG. Role of obesity in the development of osteoarthrosis and concomitant diseases. Ter Arkh. 2010;82(10):34-37. Russian.

12. Hopman WM, Berger C, Joseph L, et al. The association between body mass index and health-related quality of life: data from CaMos, a stratified population study. Qual Life Res. 2007;16(10): 1595-1603.

13. Health Canada. Canadian guidelines for body weight classification in adults. Ottawa, Canada: Health Canada Publications Centre; 2003. Report No: 4645.

14. World Health Organization. Obesity: preventing and managing the global epidemic. Report of a WHO Consultation on Obesity. Geneva, Switzerland: World Health Organization; 2000.

15. Niu J, Zhang YQ, Torner J, et al. Is obesity a risk factor for progressive radiographic knee osteoarthritis? Arthritis Rheum. 2009;61(3): 329-335.

16. Wang Y, Wluka AE, Simpson JA, et al. Body weight at early and middle adulthood, weight gain and persistent overweight from early adulthood are predictors of the risk of total knee and hip replacement for osteoarthritis. Rheumatology (Oxford). 2013;52(6): 1033-1041.

17. Blagojevic M, Jinks C, Jeffery A, Jordan KP. Risk factors for onset of osteoarthritis of the knee in older adults: a systematic review and meta-analysis. Osteoarthritis Cartilage. 2010;18(1):24-33. 
18. Sharma L, Kapoor D, Issa S. Epidemiology of osteoarthritis: an update. Curr Opin Rheumatol. 2006;18(2):147-156.

19. Zhang Y, Jordan JM. Epidemiology of osteoarthritis. Rheum Dis Clin North Am. 2008;34(3):515-529.

20. Ackerman IN, Osborne RH. Obesity and increased burden of hip and knee joint disease in Australia: results from a national survey. BMC Musculoskelet Disord. 2012;13:254.

21. Segal NA, Yack HJ, Khole P. Weight, rather than obesity distribution, explains peak external knee adduction moment during level gait. $\mathrm{Am} \mathrm{J}$ Phys Med Rehabil. 2009;88(3):180-188.

22. Christensen R, Bartels EM, Astrup A, Bliddal H. Effect of weight reduction in obese patients diagnosed with knee osteoarthritis: a systematic review and meta-analysis. Ann Rheum Dis. 2007;66(4):433-439.

23. Sridhar MS, Jarrett CD, Xerogeanes JW, Labib SA. Obesity and symptomatic osteoarthritis of the knee. J Bone Joint Surg Br. 2012;94(4): 433-440.

24. Talbot LA, Gaines JM, Huynh TN, Metter EJ. A home-based pedometerdriven walking program to increase physical activity in older adults with osteoarthritis of the knee: a preliminary study. JAm Geriatr Soc. 2003;51(3):387-392.

25. Fernandes L, Hagen KB, Bijlsma JW, et al. EULAR recommendations for the non-pharmacological core management of hip and knee osteoarthritis. Ann Rheum Dis. 2013;72(7):1125-1135.

26. Penninx BW, Messier SP, Rejeski WJ, et al. Physical exercise and the prevention of disability in activities of daily living in older persons with osteoarthritis. Arch Intern Med. 2001;161(19):2309-2316.

27. Eastlack RK, Hargens AR, Groppo ER, Steinbach GC, White KK, Pedowitz RA. Lower body positive-pressure exercise after knee surgery. Clin Orthop Relat Res. 2005;(431):213-219.

28. Quigley EJ, Noh H, Groppo ER. Gait mechanics using a lower body positive pressure chamber for orthopaedic rehabilitation. Trans Orthop Res Soc. 2000;25:828.

29. Hargens A, Cutuk A, White K. Cardiovascular impact of lower body positive pressure. Med Sci Sports Exerc. 1999;31(1S)

30. Cutuk A, Groppo ER, Quigley EJ, White KW, Pedowitz RA, Hargens AR. Ambulation in simulated fractional gravity using lower body positive pressure: cardiovascular safety and gait analyses. $J$ Appl Physiol. 2006;101(3):771-777.

31. Kurz MJ, Corr B, Stuberg W, Volkman KG, Smith N. Evaluation of lower body positive pressure supported treadmill training for children with cerebral palsy. Pediatr Phys Ther. 2011;23(3):232-239.

32. Ruckstuhl H, Kho J, Weed M, Wilkinson MW, Hargens AR. Comparing two devices of suspended treadmill walking by varying body unloading and Froude number. Gait Posture. 2009;30(4):446-451.

33. Grabowski AM, Kram R. Effects of velocity and weight support on ground reaction forces and metabolic power during running. $J \mathrm{Appl}$ Biomech. 2008;24(3):288-297.

34. Liebenberg J, Scharf J, Forrest D, Dufek JS, Masumoto K, Mercer JA. Determination of muscle activity during running at reduced body weight. J Sports Sci. 2011;29(2):207-214.

35. Patil S, Steklov N, Bugbee WD, Goldberg T, Colwell CW Jr, D'Lima DD. Anti-gravity treadmills are effective in reducing knee forces. J Orthop Res. 2013;31(5):672-679.
36. Gojanovic B, Cutti P, Shultz R, Matheson GO. Maximal physiological parameters during partial body-weight support treadmill testing. Med Sci Sports Exerc. 2012;44(10):1935-1941.

37. Takacs J, Leiter JR, Peeler JD. Novel application of lower body positive-pressure in the rehabilitation of an individual with multiple lower extremity fractures. J Rehabil Med. 2011;43(7):653-656.

38. Kellgren JH, Lawrence JS. Radiological assessment of osteo-arthrosis. Ann Rheum Dis. 1957;16(4):494-502.

39. Wendel-Vos GC, Schuit AJ, Saris WH, Kromhout D. Reproducibility and relative validity of the short questionnaire to assess health-enhancing physical activity. J Clin Epidemiol. 2003;56(12):1163-1169.

40. Roos EM, Lohmander LS. The Knee Injury and Osteoarthritis Outcome Score (KOOS): from joint injury to osteoarthritis. Health Qual Life Outcomes. 2003;1:64.

41. Roos EM, Roos HP, Lohmander LS, Ekdahl C, Beynnon BD. Knee Injury and Osteoarthritis Outcome Score (KOOS): development of a self-administered outcome measure. J Orthop Sports Phys Ther. 1998;28(2):88-96.

42. Roos EM. Joint injury causes knee osteoarthritis in young adults Curr Opin Rheumatol. 2005;17(2):195-200.

43. Gelber AC, Hochberg MC, Mead LA, Wang NY, Wigley FM, Klag MJ. Joint injury in young adults and risk for subsequent knee and hip osteoarthritis. Ann Intern Med. 2000;133(5):321-328.

44. Neuman P, Englund M, Kostogiannis I, Friden T, Roos H, Dahlberg LE. Prevalence of tibiofemoral osteoarthritis 15 years after nonoperative treatment of anterior cruciate ligament injury: a prospective cohort study. Am J Sports Med. 2008;36(9):1717-1725.

45. Louboutin H, Debarge R, Richou J, et al. Osteoarthritis in patients with anterior cruciate ligament rupture: a review of risk factors. Knee. 2009;16(4):239-244.

46. Lohmander LS, Englund PM, Dahl LL, Roos EM. The long-term consequence of anterior cruciate ligament and meniscus injuries: osteoarthritis. Am J Sports Med. 2007;35(10):1756-1769.

47. Browning RC, Kram R. Effects of obesity on the biomechanics of walking at different speeds. Med Sci Sports Exerc. 2007;39(9): 1632-1641.

48. Messier SP, Loeser RF, Miller GD, et al. Exercise and dietary weight loss in overweight and obese older adults with knee osteoarthritis: the Arthritis, Diet, and Activity Promotion Trial. Arthritis Rheum. 2004;50(5):1501-1510.

49. Altman RD, Hochberg M, Moskowitz RW, Schnitzer TJ. Recommendations for the medical management of osteoarthritis of the hip and knee. Arthritis Rheum. 2000;43(9):1905-1915.

50. Pelland L, Brosseau L, Wells G, et al. Efficacy of strengthening exercises for osteoarthritis (part I): a meta-analysis. Physical Therapy Reviews. 2004;9(2):77-108

51. Roddy E, Zhang W, Doherty M. Aerobic walking or strengthening exercise for osteoarthritis of the knee? A systematic review. Ann Rheum Dis. 2005;64(4):544-548.
Clinical Interventions in Aging

\section{Publish your work in this journal}

Clinical Interventions in Aging is an international, peer-reviewed journal focusing on evidence-based reports on the value or lack thereof of treatments intended to prevent or delay the onset of maladaptive correlates of aging in human beings. This journal is indexed on PubMed Central, MedLine, the American Chemical Society's 'Chemical Abstracts Ser-

\section{Dovepress}

vice' (CAS), Scopus and the Elsevier Bibliographic databases. The manuscript management system is completely online and includes a very quick and fair peer-review system, which is all easy to use. Visit http://www.dovepress.com/testimonials.php to read real quotes from published authors. 